\title{
Weighting Method to Identify Interharmonics based on Calculating the Bandwidth in Group-Harmonics
}

\author{
Hani Vahedi ${ }^{\dagger, * *}$, Alireza Alizadeh Kiapi ${ }^{*}$, Mohammad Tavakoli Bina ${ }^{*}$, and Kamal Al-Haddad ${ }^{* *}$ \\ ${ }^{\dagger}$ Islamic Azad University, Sari Branch, Sari, Iran \\ *Faculty of Electrical and Computer Engineering, Khajeh Nasir Toosi Univercity of Tehnology, Tehran, Iran \\ ${ }^{* *}$ Ecole de Technologie Superieure (ETS), University of Quebec, Montreal, Canada
}

\begin{abstract}
Power converters produce a vast range of harmonics, subharmonics and interharmonics. Harmonics analyzing tools based on the Fast Fourier Transform (FFT) assume that only harmonics are present and the periodicity intervals are fixed, while these periodicity intervals are variable and long in the presence of interharmonics. Using FFT may lead to invalid and undesired results due to the above mentioned issues. They can also lead to problems such as frequency blending, spectral leakage and the picket-fence effect. In this paper, the group-harmonic weighting (GHW) approach has been presented to identify the interharmonics in a power system. Afterwards, a modified GHW has been introduced to calculate the proper bandwidth for analyzing the various values of interharmonics. Modifying this method leads to more precise results in the FFT of a waveform containing inter harmonics especially in power systems with a fundamental frequency drift or frequency interference. Numerical simulations have been performed to prove the efficiency of the presented algorithm in interharmonics detection and to increase the accuracy of the FFT and the GWH methods.
\end{abstract}

Key words: Fast Fourier Transform, GHW Bandwidth Calculation, Group-Harmonic Weighting, Interharmonics

\section{INTRODUCTION}

One of the important issues in terms of delivered power quality to consumers is power system harmonics. Quick advances in power electronic semiconductors and the use of nonlinear loads in industry will increase the harmonics in current and voltage waveforms, consequently [1].

For example, frequency converters produce a vast range of harmonics components that reduce the delivered energy quality and increase power losses with low reliability in power systems that result in the need for filtering devices [2], [3].

Interharmonics and subharmonics cannot be avoided in a harmonics' debate. Interharmonics refers to the proportion of a system's main frequency located between two harmonics. In other words, interharmonics are non-integer multiplies of the main harmonic component. For instance, in a waveform where the main harmonics frequency is $50 \mathrm{~Hz}$, the harmonic

Manuscript received Jan. 22, 2012; revised Nov. 30, 2012

Recommended for publication by Associate Editor Kyo-Beum Lee.

†Corresponding Author: haaaaaaniiiiii@gmail.com

Tel: +98-151-4465-8584, Islamic Azad University

*Faculty of Electrical and Computer Engineering, Khajeh Nasir Toosi

Univercity of Tehnology, Iran

${ }^{* *}$ Ecole de Technologie Superieure (ETS), University of Quebec, Canada components with frequencies of $72.3 \mathrm{~Hz}, 90 \mathrm{~Hz}$ and $125.7 \mathrm{~Hz}$ are interharmonics. Subharmonics are also a kind of interharmonics but with a frequency that is lower than the main frequency, like $40 \mathrm{~Hz}$ [4].

Accurate measurement of the harmonics in a waveform is important in order to control and protect a system or to design suitable filters to eliminate them. As a result, their identification is vital. However, various methods such as least squares, Kalman filtering and artificial neural networks have been used to identify the harmonics in a waveform [5]-[7]. Power quality detection methods are mainly based on a FFT that is fast and simple in implementation. Although, all of the mentioned methods have their own advantages, none of them has appropriate performance in detecting interharmonics especially with a drift in the fundamental frequency of a power network in real systems.

Lin presented a GHW method based on the FFT [8]. In this case, around the main frequency, a bandwidth $(\tau)$ is considered to perform the FFT in an imaginary band. According to the numerical examples, a bandwidth obtained through trial and error and a good approximation of the values of the amplitude and frequency of an interharmonic are achieved in both the cases in which the main frequency is fixed and when it has a drift. In this paper, a novel algorithm 
is presented to calculate the bandwidth efficiently and optimally. By using this method, the value of the bandwidth used in the GHW approach is calculated through an optimum algorithm which leads to operation at deferent frequencies and being expandable.

In this paper, the GHW method is described in section II. The proposed algorithm to calculate the bandwidth is explained in section III. Numerical simulations have been done in the MATLAB environment to validate this proposed algorithm. The results are shown in section IV.

\section{Group-Harmonic Weighting (GHW)}

As shown in [9], suppose the waveform $i_{s}(t)$ is sampled at $\mathrm{N}$ discrete points using a sampling rate of $f_{s}$ where the truncation interval is $T=\frac{N}{f_{s}}$. By using digital signal processing (DSP), the continuous signal $i_{s}(t)$ can be converted into a discrete signal $i_{s}[n]$ and can be transformed by the Discrete Fourier Transform (DFT) as follows:

$$
I_{s}[k]=\frac{1}{N} \sum_{n=0}^{N-1} i_{s}[n] W_{N}^{k n}
$$

where $I_{s}[k]$ denotes the DFT of $i_{s}[n]$ at a frequency of $f_{k}$ where $f_{k}=\frac{k}{T}$ and $W_{N}=e^{\frac{j 2 \pi}{N}}$. The inverse DFT which recovers the signal from its spectrum is given by:

$$
i_{s}[n]=\sum_{k=0}^{\frac{N}{2}-1} I_{s}[k] W_{N}^{-k n}
$$

By the Parseval relation in its discrete form, the power of the waveform, $P$, can be expressed as follows:

$$
P=\frac{1}{N} \sum_{n=0}^{\frac{N}{2}-1} i_{s}[n]^{2}=\sum_{k=0}^{\frac{N}{2}-1} I_{s}[k]^{2}
$$

Therefore, the power at discrete frequency $f_{k}$ can be expressed as:

$$
P\left[f_{k}\right]=I_{s}[k]^{2}+I_{s}[N-k]^{2}=2 I_{s}[k]^{2}
$$

where $k=0,1,2, \ldots, N / 2-1$.

The RMS value of the harmonic amplitude at discrete frequency $f_{k}$ is:

$$
I_{h}\left[f_{k}\right]=\sqrt{P\left[f_{k}\right]}=\sqrt{2} I_{s}[k]
$$

Due to spectral leakage, the power of the harmonic at $f_{k}$ may disperse over the frequency band around the $f_{k}$. Hence the total harmonic power can be shown by a group in the frequency next to $f_{k}$. Each group power $\left(P^{*}\left[f_{k}\right]\right)$, is the total power between $f_{k-\Delta k}$ and $f_{k+\Delta k}$. It is described as:

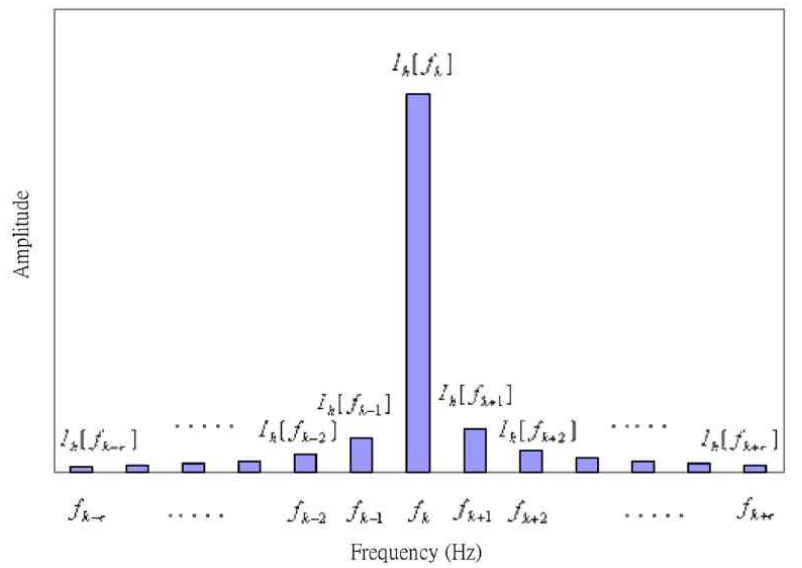

Fig. 1. Frequency spectrum of interharmonics for small frequency deviation.

$$
P^{*}\left[f_{k}\right]=\sum_{\Delta k=-\tau}^{+\tau} I_{h}\left[f_{k+\Delta k}\right]^{2}
$$

$\tau$ is an integer that represents the group bandwidth. Hence each harmonic's amplitude can be estimated as follows:

$$
I_{s}^{*}\left[f_{k}\right]=\sqrt{P^{*}\left[f_{k}\right]}
$$

By calculating a group power, the problems related to the spectral leakage will be solved. These problems are caused by measuring the interharmonics in a power system with a frequency drift. When more leakages can be gathered and considered in a group, it looks like they are in the dominant harmonic frequency. As a result, the amplitude of the interharmonics and subharmonics can be determined using this method [10].

\section{A. Group-Harmonic Weighting Model using the Bandwidth}

At first, this model divides interharmonics into two parts which means "small frequency deviation" and "large frequency deviation" in decimal points [8]. Small frequency deviation includes 0.1 to $0.5 \mathrm{~Hz}$ in decimal points. On the other hand, large frequency deviation is more than $0.5 \mathrm{~Hz}$ in decimal points. Interharmonics are shown in Fig. 1 and 2 for small and large frequency deviations. Based on spectral analysis by the FFT for small frequency deviation (less or equal to $0.5 \mathrm{~Hz}$ ), the second largest amplitude is on the right side of the dominant amplitude (Fig. 1). The second largest amplitude for the large frequency deviation (more than 0.5 $\mathrm{Hz}$ ) is on the left side of the dominant amplitude (Fig. 2).

An interharmonic frequency is described as the total center frequency $f_{k}$ plus the frequency deviation ratio (F.D.R.) which means that $f_{k}+\Delta f_{k}$ and that $\Delta f_{k}$ is used in calculating the F.D.R.

$$
F . D . R .\left(=\Delta f_{k}\right) \equiv \frac{\sqrt{\sum_{\Delta k=1}^{+\tau} I_{h}\left[f_{k+\Delta k}\right]^{2}}}{\sqrt{\sum_{\Delta k=-\tau}^{0} I_{h}\left[f_{k+\Delta k}\right]^{2}}+\sqrt{\sum_{\Delta k=1}^{+\tau} I_{h}\left[f_{k+\Delta k}\right]^{2}}}
$$




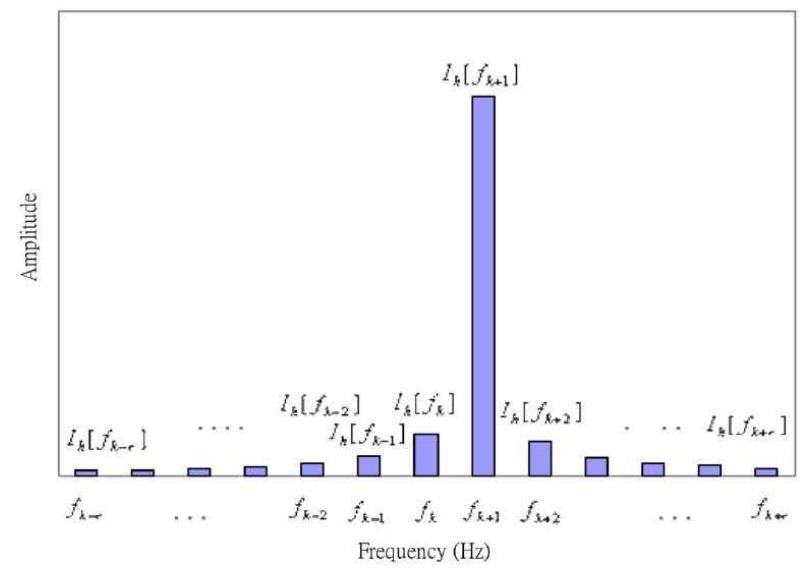

Fig. 2. Frequency spectrum of interharmonics for large frequency deviation.

Overall, the system frequency can be moved in real networks. This means that the system frequency is not exactly $50 \mathrm{~Hz}$ (e.g. $50 \mathrm{~Hz}$ is used in this paper) and that it may be more $(50.2 \mathrm{~Hz})$ or less $(49.9 \mathrm{~Hz})$. Therefore, the restored amplitude (R.A.) which is the interharmonic restored amplitude is defined as:

$$
R . A .=\frac{\sqrt{\sum_{\Delta k=-\tau}^{+\tau} I_{h}\left[f_{k+\Delta k}\right]^{2}}}{R \cdot A \cdot S \cdot F .}
$$

The R.A.S.F. is the restored amplitude in the system frequency and $f_{k}$ is the interharmonic central frequency.

$$
R \cdot A . F .=\sqrt{\sum_{\Delta l=-\tau}^{+\tau} I_{h}\left[f_{l+\Delta l}\right]^{2}}
$$

The different frequency analysis and the interharmonic amplitude are studied and discussed using the FFT and the GHW. This is easy to show. First, only one harmonic component is injected into the main waveform $(50 \mathrm{~Hz}$ and amplitude 1) and then it is surveyed under the FFT and the GHW.

$$
i_{a}=A_{a} \sin \left(2 \pi f_{a} t+\varphi_{a}\right)
$$

For this purpose, the harmonic waveform in equation (11) with different deviations (small and large) is added to the original waveform, and then the FFT analysis of this waveform is shown in figures. In addition, by using the GHW method, the values of the F.D.R. and the R.A. are calculated and compared with the FFT results. It should be mentioned that, in the GHW method, the value of the bandwidth is considered as $\tau=4$ which is recommended in [8] as the best bandwidth value.

\section{B. Without a System Frequency Drift (The system frequency has no movement)}

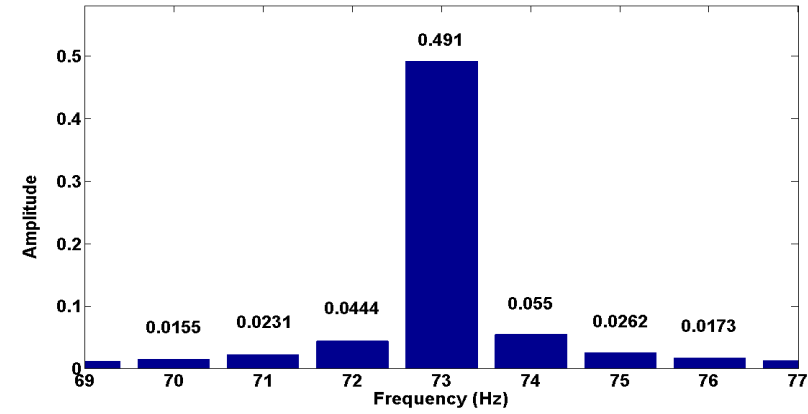

Fig. 3. FFT analysis at $73.1 \mathrm{~Hz}$ without system frequency drift.

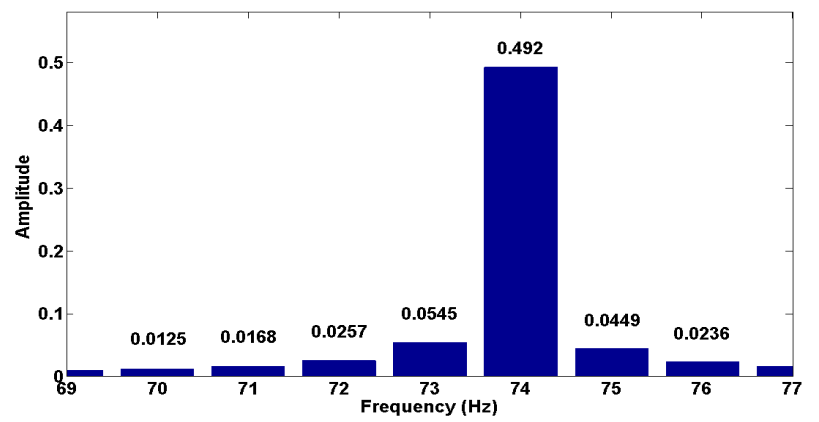

Fig. 4. FFT analysis at $73.9 \mathrm{~Hz}$ without system frequency drift.

The system frequency, i.e. $50 \mathrm{~Hz}$, is considered to be ideal and its amplitude has been normalized to 1 in following examples:

Case 1: Small Frequency Deviation, $f_{a}=73.1 \mathrm{~Hz}, \varnothing=0^{\circ}$, $A_{a}=0.5$

The FFT result is shown in Fig. 3. Using equation 8 in the GHW the F.D.R. is calculated for more than $73 \mathrm{~Hz}$ as:

F.D.R. $=0.1156 \approx 0.1$

Therefore, in this case, the interharmonic frequency is $73+0.1=73.1 \mathrm{~Hz}$ which matches the actual frequency. The R.A. is computed by equation 9 :

$$
\text { R.A. }=0.4986 \approx 0.5
$$

The interharmonic amplitude is almost 0.5 which is near to the actual one.

As can be seen in Fig. 3, the decimal point of the frequency is not included in the FFT result, while using the GHW leads to an exact interharmonic frequency. Since the frequency deviation is small, the obtained frequency in the FFT is a small integer close to the real value (i.e. $73 \mathrm{~Hz}$ ).

Case 2: Large Frequency Deviation, $f_{a}=73.9 \mathrm{~Hz}, \varnothing=0^{\circ}$, $A_{a}=0.5$

The FFT analysis is shown in Fig. 4. The F.D.R. and the R.A. can be calculated using equations 8 and 9:

F.D.R. $=0.8846 \approx 0.9$

R.A. $=0.4990 \approx 0.5$

The interharmonic frequency while using the GHW is equal to $73+0.9=73.9 \mathrm{~Hz}$. These results including the F.D.R. and the R.A. are near to the real ones.

By observing Fig. 4, it can be seen that because of the large frequency deviation, the frequency obtained in the FFT is an integer value that is larger than the actual frequency. 
Using the GHW, results in a precise value for the interharmonic frequency.

The harmonic amplitude in the FFT and the GHW are same with a small difference when the system frequency is unchanged.

\section{With a System Frequency Drift (The system frequency has a small movement)}

The main frequency has been normalized with a frequency deviation to about $50.2 \mathrm{~Hz}$ and an amplitude of 1 . The spectrum analysis result using the FFT in this frequency is shown in Fig. 5.

The computed F.D.R. and R.A. are as follows:

F.D.R. $=0.201 \approx 0.2$

R.A. $=1.064$

In this case, the system frequency obtained is equal to 50.2 $\mathrm{Hz}$ which is the same as actual frequency. It can be seen that the amount of R.A. is increased to about 0.064 in comparison with the actual amplitude which is caused by an inappropriate bandwidth. In this way, the R.A. needs to be corrected when the system frequency has a movement or change. To correct this issue, first of all, the R.A.S.F. of that movement should be calculated and then the R.A. obtained for each spectrum must be divided into the R.A.S.F. and reach the modified R.A. On the other hand, the F.D.R. calculation is accurate without any correction. It should be mentioned that the greater the main frequency deviation, the greater the value of the R.A.S.F. will be.

To show the inaccuracy of the FFT in cases with a system frequency drift, Case 1 in section II.1.1 has been considered under the new condition with a main frequency change (50.2 $\mathrm{Hz}$ ).

Fig. 6 shows the FFT result. The F.D.R. and the R.A. are as follows:

F.D.R. $=0.1411 \approx 0.1$

R.A. $=0.4905 \approx 0.5$

As can be seen, the FFT and GHW results have a lot of differences due to the main frequency drift. This issue is one of the FFT method limitations. But the GHW could calculate the values more accurately. It should be mentioned that the greater the bandwidth value, the greater the accuracy in the R.A. of the interharmonics. However, this may cause some frequency interference with the neighbouring harmonics which can lead to inaccurate results of the F.D.R.. As mentioned above, $\tau=4$ is recommended in [8] and used in this paper.

\section{BANDWIDTH CALCULATION}

The other limitation of the GHW is its frequency interference. This means that when a current or voltage signal has interharmonics near a harmonic, it may cause some interference leads to inappropriately identify the interharmonics' frequency and amplitude. Assume that $y(t)$ is a voltage or current signal:

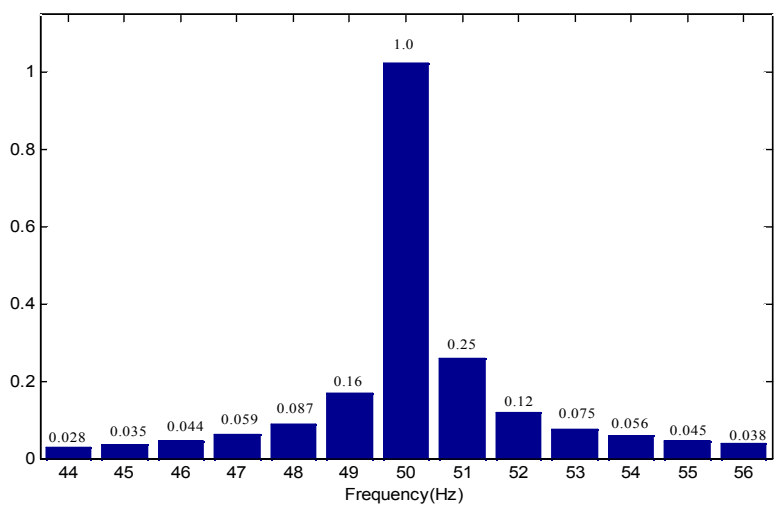

Fig. 5. Spectrum analysis at $50.2 \mathrm{~Hz}$.

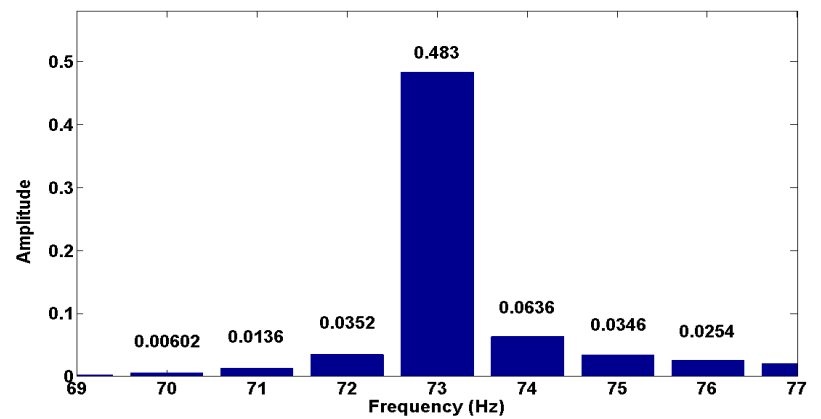

Fig. 6. FFT analysis at $73.9 \mathrm{~Hz}$ with system frequency drift.

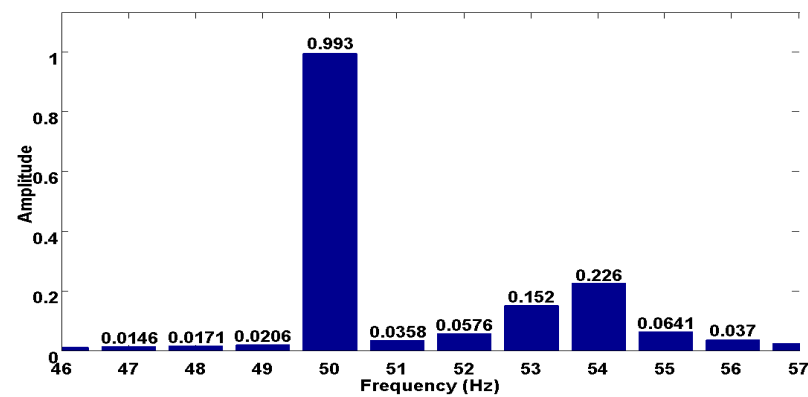

Fig. 7. FFT analysis at $53.6 \mathrm{~Hz}$.

$$
y(t)=A_{1} \cos \left(2 \pi f_{1} t\right)+A_{2} \cos \left(2 \pi f_{2} t\right)
$$

where the main component includes $f_{1}=50 \mathrm{~Hz}$ and $A_{1}=1$ and the interharmonic has a frequency of $f_{2}=53.6 \mathrm{~Hz}$ and an amplitude of $A_{2}=0.3$.s

Fig. 7 contains the FFT result of $y(t)$. To analysis a signal using the GHW, $\tau=4$ has been chosen as recommended in [8].

The F.D.R. and the R.A. for two harmonics and interharmonics are as follows:

$$
\begin{aligned}
& \text { F.D.R. }{ }_{1}=0.201 \approx 0.2205 \\
& \text { R.A. }{ }_{1}=1.0323 \\
& \text { F.D.R. } ._{2}=0.201 \approx 0.1921 \\
& \text { R.A. }{ }_{2}=1.0350
\end{aligned}
$$

As can be seen in Fig. 7, the FFT method is able to approximately calculate the interharmonic amplitude, but as before, it is unable to obtain the exact frequency of the interharmonic. The main point is the GHW method's defect in computing interharmonic's frequency and amplitude. As can be seen from the calculations, these amounts are completely wrong and make the accuracy of this method doubtful. 


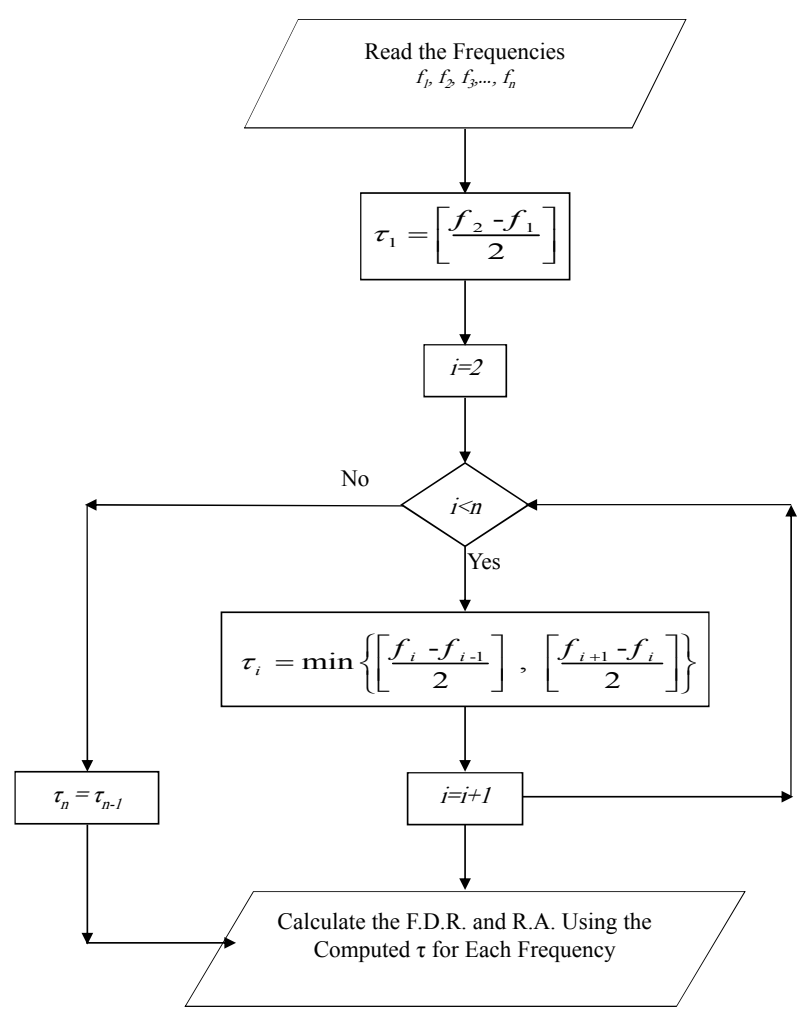

Fig. 8. Proposed algorithm to calculate the bandwidth in GHW

By analyzing the recommended bandwidth, it is realized that the frequencies used in this part are mixed together.

$$
46<f_{1}<54
$$$$
50<f_{2}<58
$$

According to these amounts, it is clear that in the interval [50], [54] Hz, frequency interference prevents the proper computation of the interharmonic frequency and amplitude. Thus, the amount of bandwidth should be decreased. The problem of interference in the frequency limit will occur while choosing a bandwidth when a subharmonic is located near a main harmonic. To remedy this issue, the bandwidth should be reduced.

In this paper, the presented algorithm calculates a suitable bandwidth for each frequency. It assumed that the frequencies of the main component and the harmonic components of a waveform are $f_{1}, f_{2}, f_{3}, \ldots, f_{n}$, respectively. From Fig. 8, it can be seen that the proposed algorithm can calculate the bandwidth for the frequency of the harmonics, interharmonics and subharmonics separately.

It can be seen in Fig. 8, that by using the proposed algorithm, the bandwidth can be calculated for each number of harmonics. It can also be seen that the amount of bandwidth is large enough to achieve a closer approximation of the harmonic amplitude. This value of the bandwidth is not only big enough to cover a vast range of group harmonics, but also small enough to prevent frequency interference.

\section{SIMULATION AND COMPARISON}

In this section numerical simulations have been done using MATLAB to validate the presented algorithm. These simulations have been conducted on two main and two subordinate cases.

In the first main case, several waves with the different frequencies and amplitudes used in [8] are simulated. Then the two subordinate cases containing a constant main frequency and a main frequency drift are considered and the results are gathered. In the second main case, several waves with different frequencies and amplitudes which have frequency interference are used and the two subordinate cases are imposed on them. The results show that the proposed algorithm efficiency in calculating the most proper bandwidth leads to greater accuracy in the frequency and amplitude of the interharmonics.

\section{A. Using Waveforms with High Frequency Differences}

In this subsection, some waves with the characteristics used in [7] have been studied and are shown below:

$$
\begin{aligned}
s(t) & =0.3 \cos \left(2 \pi f_{1} t+90^{\circ}\right) \\
& +1.0 \cos \left(2 \pi f_{2} t\right) \\
& +0.4 \cos \left(2 \pi f_{3} t+40^{\circ}\right) \\
& +0.5 \cos \left(2 \pi f_{4} t+70^{\circ}\right) \\
& +0.2 \cos \left(2 \pi f_{5} t+50^{\circ}\right) \\
& +0.1 \cos \left(2 \pi f_{6} t+80^{\circ}\right)
\end{aligned}
$$

The frequency values are shown in Table I.

TABLE I

FREQUENCIES' VALUES IN EQUATION 13

\begin{tabular}{|c|c|}
\hline$f_{1}$ & $19.2 \mathrm{~Hz}$ \\
\hline$f_{2}$ & $50.0 \mathrm{~Hz}$ \\
\hline$f_{3}$ & $98.7 \mathrm{~Hz}$ \\
\hline$f_{4}$ & $250.0 \mathrm{~Hz}$ \\
\hline$f_{5}$ & $350.0 \mathrm{~Hz}$ \\
\hline$f_{6}$ & $450.0 \mathrm{~Hz}$ \\
\hline
\end{tabular}

TABLE II

AMPLITUDES AND FREQUENCIES OF HARMONICS IN $S(T)$ WITHOUT System FREQUENCY DRIFT USING FREQUENCIES IN TABLE I

\begin{tabular}{|c|c|c|c|}
\hline & Actual Value & Using $\tau=4$ & $\begin{array}{c}\text { Using proposed } \\
\text { algorithm }\end{array}$ \\
\hline R.A.1 & 0.3 & 0.2981 & 0.3001 \\
\hline F.D.R. 1 & 0.2 & 0.2184 & 0.2249 \\
\hline R.A.2 & 1.0 & 0.9971 & 0.9972 \\
\hline F.D.R.2 & 0 & 0.0069 & 0.0140 \\
\hline R.A.3 & 0.4 & 0.3936 & 0.3986 \\
\hline F.D.R. 3 & 0.7 & 0.6784 & 0.6733 \\
\hline R.A.4 & 0.5 & 0.5002 & 0.5002 \\
\hline F.D.R.4 & 0 & 0.0014 & 0.0040 \\
\hline R.A.5 & 0.2 & 0.2001 & 0.2001 \\
\hline F.D.R. 5 & 0 & 0.0015 & 0.0047 \\
\hline R.A.6 & 0.1 & 0.1000 & 0.1000 \\
\hline F.D.R. 6 & 0 & 0.0018 & 0.0061 \\
\hline
\end{tabular}


Now, when the system main frequency is fixed $(50 \mathrm{~Hz})$, the numerical simulations are done while employing $\tau=4$.

As can be seen in tables II and III, in a case where there is no frequency interference, the bandwidth recommended in [8] and calculated by the proposed algorithm leads to almost identical results. In some instances, the proposed bandwidth has more accurate performance.

Table III shows the results under system frequency drift $(50.2 \mathrm{~Hz})$.

\section{B. Using Waveforms with a low Frequency Differences to Model Frequency Interference}

In this part, the waves in equation 12 are simulated through the proposed algorithm and the GHW to compare these methods in the frequency interference model.

As can be seen in tables IV and V, when there is a frequency interference, the amount of bandwidth $\tau=4$ leads to poor results that are different from the actual values. By calculating the bandwidth through the presented algorithm, the frequency interference does not occur and more accurate results are obtained in the interharmonic's frequency and amplitude. Therefore, for cases where the distance between the frequencies is more than $2 \mathrm{x} \tau+1=9$, the proposed algorithm is more efficient than using a fix value for $\tau$ (as it is taken to be 4 )

Table 5 shows the results when there is a frequency drift of $0.2(50.2 \mathrm{~Hz})$.

TABLE III

AMPLITUdES AND FREQUENCIES OF HARMONICS IN $S(T)$ WITH SYSTEM FREQUENCY DRIFT USING FREQUENCIES IN TABLE I

\begin{tabular}{|c|c|c|c|}
\hline & Actual Value & Using $\tau=4$ & Using proposed algorithm \\
\hline R.A.1 & 0.3 & 0.3022 & 0.3051 \\
\hline F.D.R.1 & 0.2 & 0.1981 & 0.2025 \\
\hline R.A.2 & 1.0 & 0.9901 & 0.9957 \\
\hline F.D.R.2 & 0.2 & 0.2231 & 0.2306 \\
\hline R.A. & 0.4 & 0.3938 & 0.4007 \\
\hline F.D.R. & 0.7 & 0.6769 & 0.6676 \\
\hline R.A.4 & 0.5 & 0.4992 & 0.4995 \\
\hline F.D.R.4 & 0 & 0.0067 & 0.0214 \\
\hline R.A.5 & 0.2 & 0.1996 & 0.2000 \\
\hline F.D.R.5 & 0 & 0.0128 & 0.0420 \\
\hline R.A.6 & 0.1 & 0.0999 & 0.1005 \\
\hline F.D.R.6 & 0 & 0.0227 & 0.0744 \\
\hline
\end{tabular}

TABLE IV

AMPLITUdES AND FREQUENCIES OF HARMONICS IN EQUATION 12 WITH FREQUENCY INTERFERENCE AND WITHOUT SYSTEM FREQUENCY DRIFT

\begin{tabular}{|c|c|c|c|}
\hline & Actual Value & Using $\tau=4$ & Using proposed algorithm \\
\hline R.A.1 & 1.0 & 1.0323 & 0.9936 \\
\hline F.D.R. ${ }_{1}$ & 0 & 0.2205 & 0.0348 \\
\hline R.A.2 & 0.3 & 1.0350 & 0.2787 \\
\hline F.D.R.2 & 0.6 & 0.1921 & 0.5816 \\
\hline
\end{tabular}

TABLE V

AMPLITUdES AND FREQUENCIES OF HARMONICS IN EQUATION 12 WITH FREQUENCY INTERFERENCE AND SYSTEM FREQUENCY DRIFT

\begin{tabular}{|c|c|c|c|}
\hline & Actual Value & Using $\tau=4$ & Using proposed algorithm \\
\hline R.A.1 & 1 & 1.0368 & 0.9836 \\
\hline F.D.R. ${ }_{1}$ & 0.2 & 0.2820 & 0.1911 \\
\hline R.A.2 & 0.3 & 1.0355 & 0.3041 \\
\hline F.D.R.2 & 0.6 & 0.2122 & 0.5779 \\
\hline
\end{tabular}

\section{CONCLUSIONS}

Identifying interharmonics using the GHW method based on the FFT has been developed to calculate the frequency and amplitude more accurately. The results verify that the GHW can be adapted with a frequency drift in the power system, which is not possible for the FFT or the DFT. Theoretically there is no limitation in location of the interharmonics, but the group bandwidth $(\tau)$ of each interharmonic should be chosen properly. Therefore, the bandwidth selection is very important. One of the GHW's defects is using the same bandwidth for all of the frequencies which has been modified by the proposed algorithm. Using the presented algorithm results in suitable values of $\tau$ for each frequency that show better results in numerical simulations, especially when the harmonics' frequencies are close to each other and may get interference.

\section{REFERENCES}

[1] E.L. Owen, "A history of harmonics in power systems," IEEE Ind. Appl. Mag., Jan./Feb. pp. 6-12, 1998.

[2] B. Singh, K. Al-haddad, A. Chandra, "A review of active filters for power quality improvement," IEEE Trans. Ind. Electron., Vol. 46, No. 5, pp. 960-971, Oct. 1999.

[3] H. Vahedi, A. Sheikholeslami, M. Tavakoli Bina, and M. Vahedi, "Review and simulation of fixed and adaptive hysteresis current control considering switching losses and high-frequency harmonics," Advances in Power Electronics, Vol. 2011, Article ID 397872, 6 pages, 2011.

[4] M. Karimi-Ghartemani and M. R. Iravani, "Measurement of harmonics/inter-harmonics of time-varying frequency," IEEE Trans. Power Del., Vol. 20, No. 1, pp.23-31, Jan. 2005 ,

[5] H. C. Lin, "Intelligent neural network based adaptive power line conditioner for real-time harmonics filtering," IEE Generation Transmission Distribution, Vol. 151, No. 5, pp.561-567, Sep. 2004.

[6] H. K. Kwok and D. L. Jones, "Improved instantaneous frequency estimation using an adaptive short-time Fourier transform," IEEE Trans. Signal Process., Vol. 48, No. 10, pp.2964-2972, Oct. 2000.

[7] J. A. Macias and A. Gomez, "Self-tuning of Kalman filters for harmonic computation," IEEE Trans. Power Del., Vol. 21, No. 1, pp.501-503, Jan. 2006.

[8] H. C. Lin, "Inter-harmonic identification using group-harmonic weighting approach based on the fft," IEEE Trans. Power Electron., Vol. 23, No.3, pp. 1309-1319, May 2008.

[9] Testing and measurement techniques: Harmonics and interharmonics: General guide on harmonics and 
interharmonics measurements and instrumentation for power supply systems and equipment connected thereto, IEC Standard 61000-4-7, 2002.

[10] S. Moo and Y. N. Chang, "Group harmonic identification in power system with non-stationary waveforms," IEE Generation Transmission Distribution, Vol. 142, No. 5, pp. 517-522, 1995.

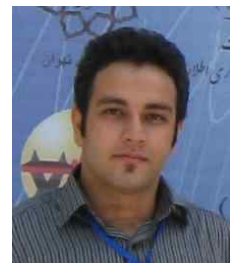

Hani Vahedi (S'10) was born in Sari, Iran, in 1986. He received his B.S. in Electrical Engineering from the K. N. Toosi University of Technology (KNTU), Tehran, Iran, in 2008, and his M.S. in Electrical Engineering from the Babol University of Technology, Babol, Iran, in 2011. He is currently pursuing his Ph.D. at the École de Technologie Superieure (ÉTS), Montreal, Canada, as a member of the Groupe de Recherche en Électronique de Puissance et Commande Industrielle (GRÉPCI). His current research interests include power quality, power electronics inverters and modulation techniques, active power filters, multilevel inverters and their application to power systems and renewable energy conversion.

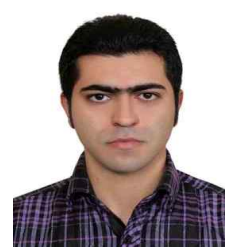

Alireza Alizadeh Kiapi was born in Sari, Iran, in 1987. He received his B.S. in Electrical Engineering from the K. N. Toosi University of Technology (KNTU), Tehran, Iran, in 2010. He is currently pursuing his M.S. in Power Electrical Engineering at the same university (KNTU). His current research interests include power quality, FACTS and power electronics applications in power systems.

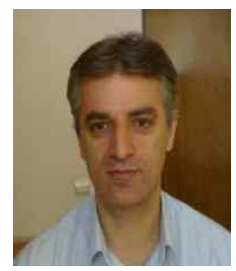

Mohammad Tavakoli Bina (SM'07) received his B.S. and M.S. from the University of Tehran, Iran, and the Ferdowsi University of Mashhad, Iran, in 1988 and 1991, respectively, and his Ph.D. from the University of Surrey, Guildford, UK, in 2001, all in Power Electronics and Power System Utility Applications. From March 1992 to November 1997, he was with the K. N. Toosi University of Technology, Tehran, Iran, as a Lecturer working on power systems. In July 2000, he received his $\mathrm{PhD}$ from the Department of Electronics and Computer Engineering, University of Surrey, UK. Since September 2001, he has been with the Faculty of Electrical and Computer Engineering, K. N. Toosi University of Technology (KNTU). He is currently a Full Professor at KNTU. His main research interests include power converters, modulation techniques, the control and modeling of FACTS controllers, distribution systems and power system control.

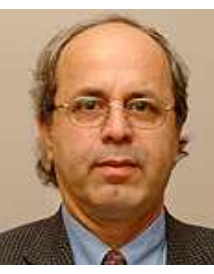

Kamal Al-Haddad (S'82-M'88-SM'92-F' 07) received his B.S. and M.S. from the University of Québec à Trois-Rivières, Trois-Rivières, QC, Canada, in 1982 and 1984, respectively, and his Ph.D. from the Institut National Polythechnique, Toulouse, France, in 1988. From June 1987 to June 1990, he was a Professor with the Engineering Department, Université du Québec à Trois Rivières. Since June 1990, he has been a Professor with the Electrical Engineering Department, École de Technologie Supérieure (ETS), Montreal, QC, where he has been the holder of the Senior Canada Research Chair in Electric Energy Conversion and Power Electronics since 2002. He has supervised more than 70 Ph.D. and M.S. students working in the field of power electronics. From 1992 to 2003 , he was the Director of the graduate study program at ETS. He is a Consultant and has established a very solid link with many Canadian industries working in the fields of power electronics, electric transportation, aeronautics, and telecommunications. He is the Chief of the ETS-Bombardier Transportation North America Division, which is a joint industrial research laboratory on electric traction systems and power electronics. He is also a coauthor of the Power System Blockset software of Matlab. He has coauthored more than 300 transactions and conference proceedings papers. His current research interests include highly efficient static power electronic converter design; harmonics and reactive power control using hybrid filters; active rectifiers and switch-mode and resonant converters, including the modeling, control, and development of prototypes for various industrial applications in electric traction, power supplies for drives, telecommunications, the chemical industry, heat induction, lighting, renewable energy converters, etc. Dr. Al-Haddad is a Fellow Member of the Canadian Academy of Engineering and a Life Member of the Circle of Excellence of the University of Quebec. He is active in the IEEE Industrial Electronics Society, where he is the Vice President for Publication, an AdCom member, and serves as an Associate Editor and a distinguished Lecturer. Dr. Al-Haddad is a member of the Order of Engineers of Quebec, Canada. 